\title{
Clinical Feasibility of Molecular Imaging of Plaque Inflammation in Atherosclerosis
}

\author{
Nobuhiro Tahara ${ }^{1,2}$, Tsutomu Imaizumi ${ }^{2}$, Renu Virmani $^{3}$, and Jagat Narula ${ }^{1}$ \\ ${ }^{1}$ University of California, Irvine, California; ${ }^{2}$ Kurume University School of Medicine, Kurume, Japan; and ${ }^{3}$ Cardiovascular \\ Pathology Institute, Gaithersburg, Maryland
}

Despite substantial advances in the diagnosis and management of coronary artery disease, acute coronary events continue to occur in many patients. It has been increasingly realized that the lesions responsible for acute events may not necessarily be critically obstructive and hence not be associated with inducible ischemia. Various morphologic features of plaque vulnerability have been described by CT angiography, intravascular ultrasound, and optical coherence tomography. The culprit plaques often demonstrate large plaque and necrotic core volumes, positive vascular remodeling, and attenuation of fibrous plaque caps. The remaining obligatory component of plaque vulnerability is fibrous cap inflammation; molecular imaging is best suited for identification of monocyte-macrophage infiltration. Whereas multiple candidate targets have been evaluated in preclinical molecular imaging studies, only ${ }^{18} \mathrm{~F}-\mathrm{FDG}$ and $99 \mathrm{mTC}$-annexin-A5 have been recently used in the settings of acute vascular events. These 2 imaging strategies have demonstrated the clinical feasibility of imaging for detection of inflammation.

Key Words: atherosclerosis; vulnerable plaque; molecular imaging; inflammation; ${ }^{18}$ F-labeled FDG PET; apoptosis; $99 \mathrm{mTC}-$ labeled annexin-A5

J Nucl Med 2009; 50:331-334

DOI: 10.2967/jnumed.108.060376

Molecular imaging of various components of atherosclerotic plaques has been proposed, and proof of principle has been demonstrated in experimental models of disease (1). These preclinical studies have predominantly targeted plaque inflammation with the premise that the extent of inflammation would determine the vulnerability of the plaque to rupture. Plaque inflammation has been detected by targeting alterations in monocytes that facilitate their migration to the neointima, ensure efficient scavenging of insudated lipid, oversee their transformation to foam cells, or mediate cell death (1). Molecular targets have also included the events that are associated with or consequent to inflammation, such as production of cytokines and metalloproteinases. Although these experimental molecular

Received Aug. 13, 2008; revision accepted Dec. 3, 2008.

For correspondence or reprints contact: Jagat Narula, University of California, UCI Medical Center, 101 The City Dr., Bldg. 53, Rt. 81, Orange, CA. E-mail: narula@uci.edu

COPYRIGHT @ 2009 by the Society of Nuclear Medicine, Inc. imaging studies have offered significant promise, translational data in the clinical setting has just started to emerge. Clinical studies of molecular targeting are the major focus of the following review. We have referred to some of the early molecular imaging attempts that labeled white blood cells to follow their localization and labeled lipoproteins to trace their destination in the inflammatory cells in plaques (1). Even though the incorporation of radiolabeled components in the plaque may not have been adequate, these studies created a sound foundation for the development of imaging strategies of the future.

\section{PATHOLOGIC BASIS OF INFLAMMATION IMAGING}

\section{Ruptured Plaques Are Substantially Inflamed}

Vulnerable plaques have typically large necrotic cores that are covered by thin fibrous caps (2). Many foam cells are seen around the necrotic cores and within the fibrous caps (Fig. 1). Pathologic examination of culprit plaques in the victims of acute coronary events reveals extensive inflammation with macrophages; the more the macrophages, the thinner the cap. Migration of monocytes to the subintimal layers of the plaque is mediated by development of receptors for chemoattractant factors such as monocyte chemotactic protein-1 (MCP-1) and those for adhesion molecules such as intercellular adhesion molecule-1 and vascular cell adhesion molecule-1 (VCAM-1) (1). After subintimal localization, the monocytes express scavenger receptors including SRAI/II, CD68, and FcRIII. In experimental models, these receptors have been targeted by radiolabeled MCP-1, VCAM-1, Fc-IgG, and lipoproteins.

\section{Inflammation Is Accompanied by Cytokine Release}

Foam cells in the neointima release numerous cytokines, such as interleukin-1, tumor necrosis factor- $\alpha$, and MCP-1, that attract other monocytes and activate endothelial cells and smooth muscle cells (3). Activated macrophages also release metalloproteinases and other proteolytic enzymes such as cathepsins, which lead to degradation of the matrix, thinning of the fibrous cap, and positive outward remodeling of the vessel wall. Activated lymphocytes produce proinflammatory cytokines such as interferon- $\gamma$, which is able to amplify the inflammatory response. Lymphokines also facilitate adventitial vasa vasorum proliferation and plaque neoangiogenesis, which contributes to red blood cell extravasation and necrotic core enlargement.

\section{Unstable Plaques Demonstrate Significant Cell Death}

Cell death is commonly observed in the vulnerable plaque; macrophage death leads to expansion of the necrotic core and perpetuates plaque instability (4). More than $40 \%$ of macrophages at the rupture site are in the process of cell death by apoptosis; 


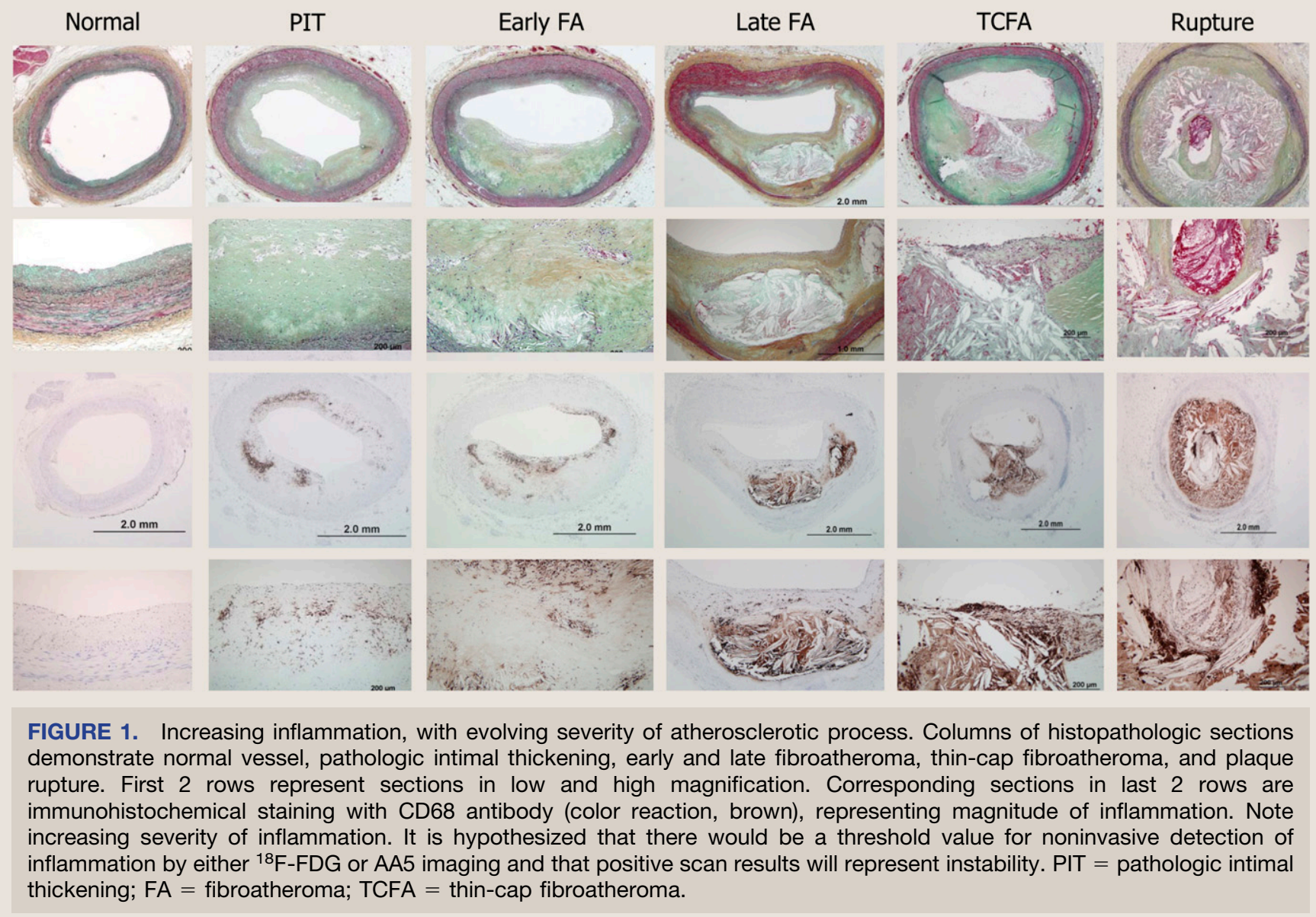

macrophages remote from the site of rupture do not show much apoptosis. It has been reported that dying smooth muscle cells may release large quantities of proinflammation cytokines such as MCP-1 and interleukin-8 (5), and dying macrophages may produce tissue factor $(6)$ and metalloproteinases.

\section{MOLECULAR IMAGING OF PLAQUE INFLAMMATION}

Numerous characteristic alterations evolve as monocytes traverse the intimal layer and prepare to ingest concurrently infiltrating lipids. These unique features, which vary based on different phases of plaque development, have been targeted successfully by radiolabeled autologous leukocytes (Fig. 2) (7), low-density lipoprotein (LDL) (8), and Fc fragments of immunoglobulin (9) for targeting of the scavenger function. More recent experimental studies have used radiolabeled ligands of cytokine and adhesion molecule receptors, including MCP-1 and VCAM-1, or cytokines released by infiltrating macrophages such as metalloproteinases (1). It has not been entirely clear if such a characterization would be of clinical significance or which candidate molecule would be most informative. However, few recent correlative studies have demonstrated that the presence of macrophages with high respiratory burst or those with activation of cell death pathways are associated with culprit lesions underlying an acute coronary event. The metabolically active macrophages have been clinically recognized by ${ }^{18} \mathrm{~F}$-labeled FDG imaging (10), and dying macrophages have been successfully targeted by using annexin-A5 (AA5) (Fig. 2) $(4,11)$. Detection of active inflammation should allow identification of vulnerable plaques if information is obtained before an acute event has occurred.

\section{${ }^{18}$ F-FDG IMAGING FOR PLAQUE INFLAMMATION}

${ }^{18}$ F-FDG Uptake in Vessels Is Commonly Seen in Patients Undergoing Evaluation of Malignant Tumors

PET imaging studies for localization of malignant tumors have reported incidental ${ }^{18} \mathrm{~F}$-FDG uptake in the carotid, coronary, iliac, and femoral arteries and thoracic and abdominal aorta; ${ }^{18} \mathrm{~F}$-FDG uptake in large arteries was observed in up to $50 \%$ of patients evaluated for oncologic reasons. In a prospective ${ }^{18} \mathrm{~F}$-FDG PET study performed in a large cohort of consecutive patients who had undergone carotid artery ultrasound imaging for screening, ${ }^{18} \mathrm{~F}$-FDG uptake was seen in $30 \%$ of patients with evidence of carotid atherosclerosis (12). Glucose uptake in atherosclerotic plaques has been hypothesized to represent inflammatory activity on the basis of cell culture studies of prominent ${ }^{18}$ F-FDG uptake by cytokine- or lipopolysaccharide-activated macrophages in parallel to the extent of cellular respiratory burst.

\section{${ }^{18}$ F-FDG Uptake in Vessels Is Related to Macrophage Infiltration}

A direct correlation between carotid ${ }^{18} \mathrm{~F}$-FDG uptake (expressed as the target-to-background ratio of standardized uptake value) and macrophage density (mean percentage staining of CD68-positive cells) in the carotid endarterectomy specimens has been prospectively demonstrated $(r=0.85, P<0.0001)(13) .{ }^{18} \mathrm{~F}$-FDG uptake did not correlate with plaque area, thickness, or smooth muscle cell density.

\section{${ }^{18}$ F-FDG Uptake Studies Allow Serial Assessment of Plaque Inflammation}

Recently, serial measurements of coronary neointimal thickening has gained significant popularity for demonstration of efficacy of 

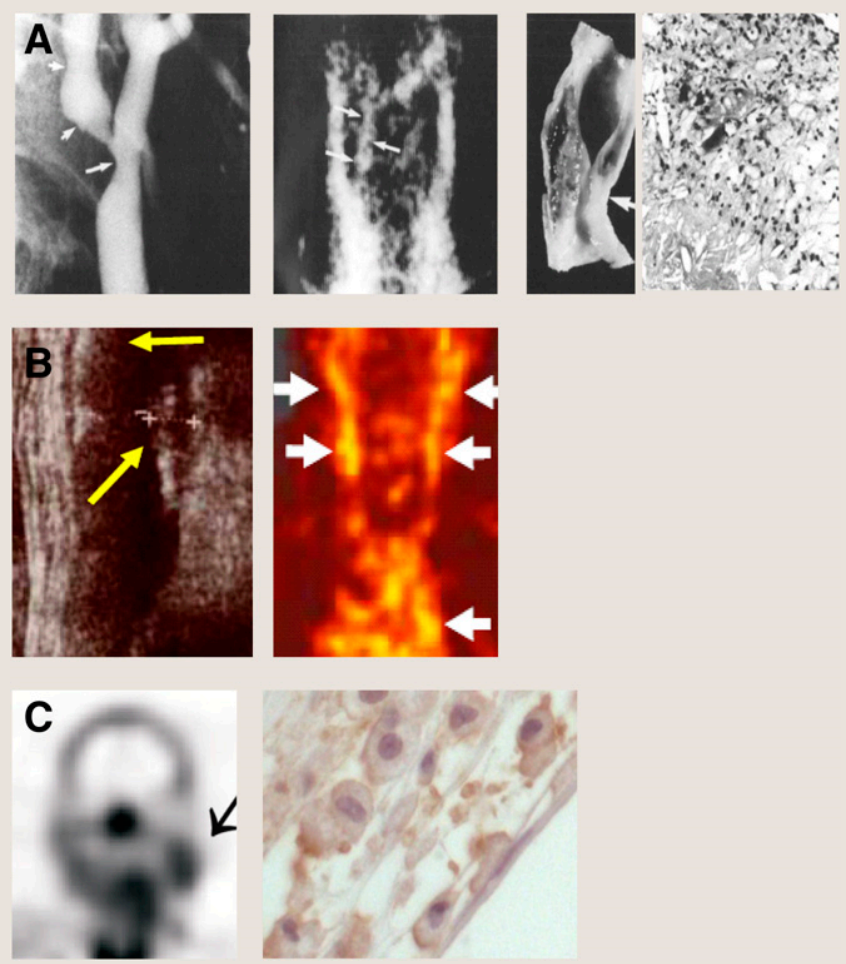

FIGURE 2. Clinical imaging of plaque inflammation. (A) Imaging of macrophage prevalence with radiolabeled LDL. Carotid angiogram shows extensive stenosis (arrow) and mural irregularities (arrowheads) involving distal common and proximal internal carotid arteries (left panel). Left carotid angiogram showed only minor degree of internal carotid stenosis. External image $9 \mathrm{~h}$ after injection of ${ }^{99 \mathrm{~m} T c-L D L}$ (481 MBq [13 mCi]) shows focal, asymmetric accumulation in right common carotid artery at bifurcation and proximal internal carotid artery (arrows), which corresponds to angiographic lesion (middle panel). Photograph of bisected right carotid endarterectomy specimen showing tight stenosis (arrow) and extensive intraplaque hemorrhage (right panel); histologic section demonstrates lesion with numerous foam cells and macrophages adjacent to hemorrhage (hematoxylin and eosin, $\times 200$ ). In clinically most feasible molecular imaging, ${ }^{18} \mathrm{~F}-\mathrm{FDG}$ is selectively taken up by infiltrating macrophages in plaques. (Image modified from (8).) (B) Carotid artery ultrasound imaging demonstrates carotid vascular disease in patient with recent acute vascular event (left panel). Plaques reveal homogeneous ultrasonic appearance, with irregular surfaces. Abnormal focal ${ }^{18} \mathrm{~F}-\mathrm{FDG}$ uptake visualized in ${ }^{18} \mathrm{~F}-\mathrm{FDG}$ PET images is concordant with ultrasonic information (right panel). (Image modified from (15).) (C) In contrast to ${ }^{18} \mathrm{~F}-\mathrm{FDG}$, annexin A5 selectively binds to dying macrophages, which are abundant in unstable plaques. Coronal SPECT view in patient with left-sided transient ischemic attack $3 \mathrm{~d}$ before surgery (left panel). Although this patient had clinically significant stenosis of both carotid arteries, annexin uptake was evident only in culprit lesion (arrow). Histopathologic analysis of endarterectomy specimen (polyclonal rabbit anti-annexin antibody, $\times 400$ ) shows substantial infiltration of macrophages into neointima, with extensive binding of AA5 (brown) (right panel). (Image modified from (11).) statin therapy and other agents likely to favorably affect atherosclerosis $(14,15)$. As such, if PET could also be used to monitor changes in plaque inflammation over time, a noninvasive tool would become available as a surrogate marker of plaque instability and the efficacy of pharmacologic interventions. Serial prospective ${ }^{18} \mathrm{~F}-\mathrm{FDG}$ PET studies have reported an excellent interobserver, intraobserver, and interscan reproducibility (14). The effect of statin intervention on ${ }^{18} \mathrm{~F}$-FDG uptake has been reported in many consecutive patients with carotid atherosclerosis (15). The follow-up PET scans revealed significant reduction in ${ }^{18} \mathrm{~F}$-FDG accumulation after statin therapy. In this study, only dietary restrictions did not show resolution of vascular inflammation.

\section{${ }^{18}$ F-FDG Imaging of Coronary Inflammation Is Feasible}

Although various case reports and retrospective studies (16) have demonstrated anecdotal ${ }^{18} \mathrm{~F}-\mathrm{FDG}$ uptake in coronary arteries in oncologic patients, a recent prospective ${ }^{18} \mathrm{~F}$-FDG PET study with multislice CT demonstrated the feasibility of precise ${ }^{18} \mathrm{~F}-\mathrm{FDG}$ localization in coronary arteries (Fig. 3) (17). In this elegant study design, myocardial ${ }^{18} \mathrm{~F}-\mathrm{FDG}$ uptake was almost entirely suppressed by a high-fat diet and restriction of carbohydrate meals for $1 \mathrm{~d}$ before the study and administration of $\beta$-blockers on the day of study. The suppression of the myocardial background facilitated better target demarcation. The study also took advantage of CT angiography and enrolled patients who had undergone coronary stent implantation for acute coronary syndrome or chronic stable angina. CT angiography and stent location allowed precise coregistration of ${ }^{18} \mathrm{~F}-\mathrm{FDG}$ uptake at the plaque site. Culprit lesions demonstrated significantly higher ${ }^{18} \mathrm{~F}$ FDG uptake (Fig. 3) than did target lesions in chronic disease. ${ }^{18} \mathrm{~F}$ FDG uptake was also prominently seen in some nonstented coronary segments and also in the aortic root. Although it will be necessary to develop measures to contain radiation burden imposed by combined PET/CT studies, this study holds a promise of radical strategic shift in coronary artery disease management.

\section{ANNEXIN IMAGING OF INFLAMED PLAQUES}

\section{The Principle and Basis of Cell Death Imaging}

Because apoptotic cells express phosphatidylserine on their cell surface and AA5 has a high affinity for binding to phosphatidylserine, imaging with ${ }^{99 \mathrm{~m}} \mathrm{Tc}$-labeled AA5 has been used to evaluate the feasibility of the detection of unstable plaques. AA5 has been extensively used previously for noninvasive imaging of experimental atherosclerotic lesions (4), and its accumulation was predominantly observed in American Heart Association-type IV lesions. There was a direct correlation of AA5 uptake with macrophage burden and the magnitude of histologically verified apoptosis. It was subsequently indicated that pharmacologic intervention using stains and caspase inhibitors could reduce the extent of apoptosis in experimental atherosclerosis models $(18,19)$. Studies of porcine atherosclerosis have demonstrated the feasibility of coronary imaging with radiolabeled AA5 (20).

\section{Annexin Uptake Is Correlated to the Extent of Cell Death in Carotid Endarterectomy Specimens}

99m Tc-AA5 has been used in a small pilot study for imaging of carotid atherosclerosis in patients with recent or remote cerebrovascular accidents (11); AA5 uptake was reported only after recent cerebrovascular accidents and not seen in patients being treated with statins. AA5 binding was histologically localized to apoptotic macrophages and also to the red blood cell membranes embedded in necrotic cores. Radiolabeling of AA5 with PET-compatible radiotracers such as ${ }^{124} \mathrm{I}$ and ${ }^{18} \mathrm{~F}$ is under way and may provide better avenues for coronary vascular imaging. 

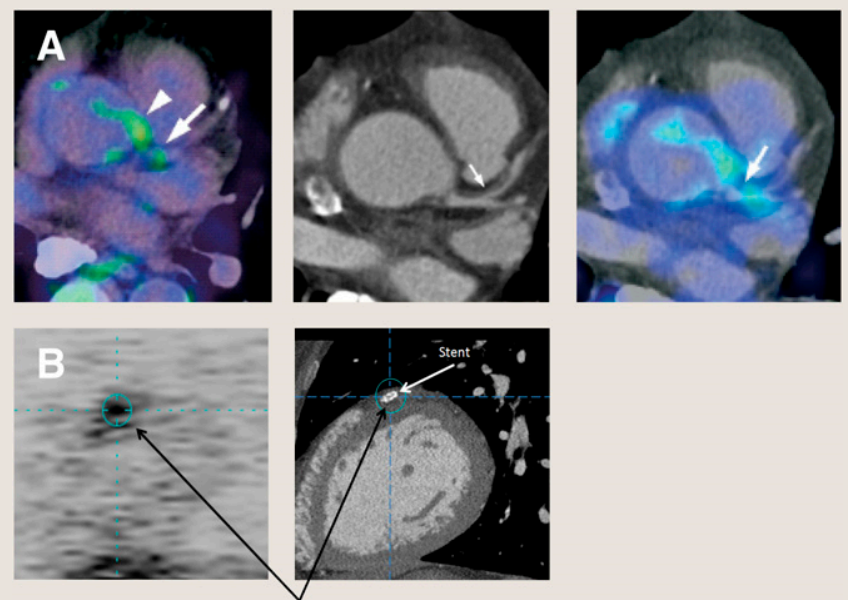

Culprit Plaque associated with recent MI

FIGURE 3. ${ }^{18} \mathrm{~F}-\mathrm{FDG}$ imaging of coronary inflammation. (A) Incidental ${ }^{18} \mathrm{~F}$-FDG uptake is seen in left main coronary artery region in 71-y-old patient undergoing PET for evaluation of recurrence of colon malignancy and metastatic disease (modified from (16)) (left panel). This patient had multiple coronary risk factor; hence, CT angiography was performed that showed noncalcified plaque in left main coronary and proximal left anterior descending artery (arrow) (middle panel). Corresponding image after fusion with ${ }^{18} \mathrm{~F}-$ FDG PET/CT localized inflammatory PET signal with maximal standardized uptake value of 2.1 (arrow) (right panel). (B) On the other hand, prospective study has recently demonstrated potential feasibility of detecting inflammation in culprit plaque in patients presenting with acute coronary syndrome. In 1 such patient who had undergone primary coronary intervention, ${ }^{18} \mathrm{~F}-\mathrm{FDG}$ imaging was performed. Radiotracer uptake is clearly visible (left) at site of coronary stent placement (right), suggesting that culprit lesion was inflamed. ${ }^{18} \mathrm{~F}-\mathrm{FDG}$ uptake in myocardium was suppressed by high-fat, low-carbohydrate diet and $\beta$-blocker administration. Stent sites in patients with chronic stable angina did not show ${ }^{18} \mathrm{~F}-\mathrm{FDG}$ uptake. $\mathrm{MI}=$ myocardial infarction. Figure 3B was provided by Ahmed Tawakol, Massachusetts General Hospital, Boston, Massachusetts.

\section{CONCLUSIONS}

The likelihood that atherosclerotic plaques will result in acute vascular events is intimately associated with the morphologic traits of the plaque and the extent of inflammation. A noninvasive strategy designed to monitor the extent of plaque inflammation may allow identification of unstable plaques, and serial interrogation may determine the efficacy of intervention. ${ }^{18}$ F-FDG uptake, which has been commonly used in oncologic practice, offers information about plaque inflammation and allows serial investigation. The feasibility of coronary imaging with ${ }^{18} \mathrm{~F}-\mathrm{FDG}$ has evoked tremendous enthusiasm in the imaging community. Successful ${ }^{18}$ F-FDG imaging of coronary arteries has also encouraged investigation with other promising molecules, such as annexin. It is conceivable that the high-risk patients identified by clinical tools, including genetic information and biomarkers, will in the future be more accurately risk-stratified by imaging targeted at morphologic and functional characterization of high-risk plaques.

\section{ACKNOWLEDGMENTS}

This work was supported in part by National Institutes of Health grants RO1 HL68657 and RO1 HL078681 and research grants from
Mitsui Life Social Welfare Foundation, Japan Heart Foundation Research, FUKUOKA clinical medicine of research prize, International Research Fund for Subsidy of Kyusyu University School of Medicine Alumni, and the Banyu Fellowship Program sponsored by Banyu Life Science Foundation International.

\section{REFERENCES}

1. Narula J, Garg P, Achenbach S, Motoyama S, Virmani R, Strauss HW. Arithmetic of vulnerable plaques for noninvasive imaging. Nat Clin Pract Cardiovasc Med. 2008;5(suppl 2):S2-S10.

2. Burke AP, Farb A, Malcom GT, Liang YH, Smialek J, Virmani R. Coronary risk factors and plaque morphology in men with coronary disease who died suddenly. N Engl J Med. 1997;336:1276-1282.

3. Libby P. Inflammation in atherosclerosis. Nature. 2002;420:868-874.

4. Kolodgie FD, Petrov A, Virmani R, et al. Targeting of apoptotic macrophages and experimental atheroma with radiolabeled annexin $\mathrm{V}$ : a technique with potential for noninvasive imaging of vulnerable plaque. Circulation. 2003;108: 3134-3139.

5. Schaub FJ, Han DK, Liles WC, et al. Fas/FADD-mediated activation of a specific program of inflammatory gene expression in vascular smooth muscle cells. Nat Med. 2000;6:790-796.

6. Hutter R, Valdiviezo C, Sauter BV, et al. Caspase-3 and tissue factor expression in lipid-rich plaque macrophages: evidence for apoptosis as link between inflammation and atherothrombosis. Circulation. 2004;109:2001-2008.

7. Virgolini I, Müller C, Fitscha P, Chiba P, Sinzinger H. Radiolabelling autologous monocytes with 111-indium-oxine for reinjection in patients with atherosclerosis. Prog Clin Biol Res. 1990;355:271-280.

8. Lees AM, Lees RS, Schoen FJ, et al. Imaging human atherosclerosis with ${ }^{99 \mathrm{~m}} \mathrm{Tc}$ labeled low density lipoproteins. Arteriosclerosis. 1988;8:461-470.

9. Fischman AJ, Rubin RH, Delvecchio A, Strauss HW. Imaging of atheromatous lesions in the iliac and femoral vessels: preliminary experience with ${ }^{111} \mathrm{In}-\mathrm{IgG}$ in human subjects [abstract]. J Nucl Med. 1989;30(suppl):817P.

10. Rudd JHF, Warburton EA, Fryer TD, et al. Imaging atherosclerotic plaque inflammation with $\left[{ }^{18} \mathrm{~F}\right]$-fluorodeoxyglucose-positron emission tomography. Circulation. 2002;105:2708-2711.

11. Kietselaer BL, Reutelingsperger CP, Heidendal GA, et al. Noninvasive detection of plaque instability with use of radiolabeled annexin A5 in patients with carotidartery atherosclerosis. $N$ Engl J Med. 2004;350:1472-1473.

12. Tahara N, Kai H, Nakaura H, et al. The prevalence of inflammation in carotid atherosclerosis: evaluation by FDG-PET. Eur Heart J. 2007;28:2243-2248.

13. Tawakol A, Migrino RQ, Bashian GG, et al. In vivo ${ }^{18} \mathrm{~F}$-fluorodeoxyglucose positron emission tomography imaging provides a noninvasive measure of carotid plaque inflammation in patients. J Am Coll Cardiol. 2006;48:1818-1824.

14. Rudd JH, Myers KS, Bansilal S, et al. ${ }^{18}$ Fluorodeoxyglucose positron emission tomography imaging of atherosclerotic plaque inflammation is highly reproducible: implications for atherosclerosis therapy trials. J Am Coll Cardiol. 2007; 50:892-896.

15. Tahara N, Kai H, Ishibashi M, et al. Simvastatin attenuates plaque inflammation: evaluation by fluorodeoxyglucose positron emission tomography. J Am Coll Cardiol. 2006;48:1825-1831.

16. Alexanderson E, Slomka P, Cheng V, et al. Fusion of positron emission tomography and coronary computed tomographic angiography identifies fluorine 18 fluorodeoxyglucose uptake in the left main coronary artery soft plaque. $\mathrm{J} \mathrm{Nucl}$ Cardiol. 2008;15:841-843.

17. Rogers IS, Figueroa AL, Nasir K, et al. Assessment of coronary segment inflammation with combined 18-fluorodeoxyglucose positron emission tomography and 64-slice multidetector computed tomography [abstract]. Circulation. 2007;116(suppl II):410.

18. Hartung D, Sarai M, Petrov A, et al. Resolution of apoptosis in atherosclerotic plaque by dietary modification and statin therapy. J Nucl Med. 2005;46:2051-2056.

19. Sarai M, Hartung D, Petrov A, et al. Broad and specific caspase inhibitorinduced acute repression of apoptosis in atherosclerotic lesions evaluated by radiolabeled annexin A5 imaging. J Am Coll Cardiol. 2007;50:2305-2312.

20. Johnson LL, Schofield L, Donahay T, Narula N, Narula J. ${ }^{99 \mathrm{~m}} \mathrm{Tc}$-annexin V imaging for in vivo detection of atherosclerotic lesions in porcine coronary arteries. J Nucl Med. 2005;46:1186-1193. 